\title{
BMJ Open Impact of secondhand smoke exposure on cognitive function among middle- aged and older women in China: findings from three waves of the China Health and Retirement Longitudinal Study
}

\author{
Anying Bai, Yinzi Jin (D) , Yangmu Huang
}

To cite: Bai A, Jin Y, Huang Y. Impact of secondhand smoke exposure on cognitive function among middle-aged and older women in China: findings from three waves of the China Health and Retirement Longitudinal Study. BMJ Open 2020;10:e039824. doi:10.1136/ bmjopen-2020-039824

\section{- Prepublication history and} additional material for this paper are available online. To view these files, please visit the journal online (http://dx.doi. org/10.1136/bmjopen-2020039824).

Received 27 April 2020 Revised 30 September 2020 Accepted 11 October 2020
Check for updates

(C) Author(s) (or their employer(s)) 2020. Re-use permitted under CC BY-NC. No commercial re-use. See rights and permissions. Published by BMJ.

School of Public Health, Peking University, Beijing, China

Correspondence to Dr Yangmu Huang; ymhuang@bjmu.edu.cn

\section{ABSTRACT}

Objectives To examine the association between secondhand smoke (SSH) and women's global cognitive function and cognitive subdomains.

Design Cohort study.

Participants Data for this study were obtained from the China Health and Retirement Longitudinal Study (2011-2013-2015), and pooled analysis was applied to wave 1 and wave 2 (2011-2013), wave 2 and wave 3 (2013-2015) and wave 1 and wave 3 (2011-2015). Data from a total of 6875 Chinese women with normal cognitive function at baseline were selected for analysis, including 2981 who were interviewed in 2011, 2471 in 2013, and 1894 in 2015.

Main outcome measures and methods SHS was classified based on the number of exposed years $(<25$ years, $\geq 25$ years to $<30$ years, $\geq 30$ years to $<40$ years, $\geq 40$ years). Global cognitive function, visuospatial ability, orientation and attention, and episodic memory function were used as measures of cognitive function. Three waves of data were pooled using a dummy variable to differentiate between 2-year and 4-year groups. LDV models were used to examine independent associations between SHS and cognitive function. Demographic factors, socioeconomic factors, baseline cognitive functioning and health conditions were controlled for in our models.

Results SSH was found to be inversely and significantly associated with cognitive function. Compared with those who had not been exposed to household SSH, women who had lived with a smoking husband had a significantly faster cognition decline, especially in global cognitive function $(\beta=-0.33,95 \% \mathrm{Cl}=-0.66$ to $-0.01, \mathrm{p}<0.01)$, visuospatial ability $(\beta=-0.04,95 \% \mathrm{Cl}=-0.08$ to -0.01 , $p<0.05)$ and episodic memory function $(\beta=-0.16,95 \%$ $\mathrm{Cl}=-0.31$ to $-0.01, \mathrm{p}=0.031$ ).

Conclusions Household SSH exposure for more than 40 years was associated with a more significant decline in global cognitive function, visuospatial ability and episodic memory function, but not in orientation and attention function among older Chinese women.
Strengths and limitations of this study

- This is the first study to investigate the association between secondhand smoke (SSH) exposure and different domains of women's cognitive function in China using 4 years of longitudinal national representative data.This study addressed the issue of reverse causation in observational cohort studies using lagged dependent variable models and adjusted for baseline cognition scores.

- The exposure to SHS was evaluated based on selfreported measures.

- The analyses only contained household SHS exposure and excluded environmental exposure.

\section{INTRODUCTION}

China's population has been ageing rapidly. By 2050, there will be 400 million Chinese citizens aged $>65$ years, 150 million of whom will be aged $>80$ years. ${ }^{1}$ It will become increasingly important to understand the cognitive changes that accompany ageing. ${ }^{2}$ Cognitive impairment, described as a decline in intellectual function, ${ }^{3}$ ranges from mild forms of forgetfulness to severe and debilitating dementia. ${ }^{4}$ The prevalence of cognitive impairment is rising, with national figures estimating that more than $9.4 \%$ of older people in China had cognitive impairment in $2011 .^{4}$

Numerous determinants, such as environmental, individual and genetic factors, could favour evolution toward cognitive impairment, and both age and late-life hypertension increase the risk of dementia over time. ${ }^{5}$ The mechanism lies in age-related functional and structural changes in cerebrovascular small and large blood vessels. ${ }^{6}$ Besides chronic disease factors, depression has long 
been known to affect memory and other neurocognitive domains. Previous studies have emphasised that depression could increase the risk of developing mild cognitive impairment (MCI) in cognitively normal elderly people. ${ }^{7}$

Exposure to secondhand smoke (SHS), also known as 'passive smoking', refers to a situation where a neversmoker inhales another person's smoke by exposure to either sidestream smoke or mainstream smoke. ${ }^{8}$ Current smoking prevalence in China has decreased from $31.1 \%$ in 2002 to $28.1 \%$ in 2010; however, the number of adults exposed to SHS during this period still increased from 540 million to 556 million due to population growth. ${ }^{2}$ The negative health effects of high levels of exposure to SHS may be close to those of active smoking, including inferior performance on measures of general intelligence, visuospatial learning and memory, and fine motor dexterity. ${ }^{9}$ Given the association between exposure to SHS and risk factors for cognitive impairment such as cardiovascular disease ${ }^{10}$ hypertension ${ }^{11}$ and stroke,${ }^{10}$ it is possible that a high level of exposure may be a preventable risk factor for cognitive impairment or dementia. ${ }^{112}$

There is some evidence to suggest that older current smokers (aged $\geq 63$ years) ${ }^{13}$ or those being exposed to SHS (aged 55-64 years) ${ }^{12-15}$ were more likely to develop cognitive impairment compared with never-smokers. However, much less is known about whether and to what extent SHS is associated with global cognitive function and cognitive subdomains among elderly women in China. Previous studies in China indicated that SHS exposure increased the risk of cognitive impairment in older adults. ${ }^{1617}$ Nevertheless, both these studies only used two-wave longitudinal data and did not control for baseline cognition. ${ }^{16}{ }^{17}$ Therefore, the primary aim of this study was to investigate the relationship between SHS and cognitive function among older non-smoking Chinese women, using three-wave longitudinal national representative data. Through the classification of respondents by different years of SHS exposure in a 4-year panel, we identified whether certain high SHS exposure groups were at higher risk of cognitive decline than others after controlling for confounders. Besides, we aimed to examine the association between SHS exposure and cognitive subdomains. This is especially important given the growing and ageing population, and increasing prevalence of SHS exposure in China.

\section{METHODS \\ Data}

China Health and Retirement Longitudinal Study (CHARLS) had passed the ethical review before field investigation, and we used data from three waves of the CHARLS (2011-2013-2015), which are publicly available at http://charls.pku.edu.cn. CHARLS involved participants with a nationally representative survey of adults aged $\geq 45$ years, as well as their spouses when possible, and included assessments of social, economic and health circumstances of community residents. The national baseline survey was conducted between June 2011 and March 2012, and samples were chosen through multistage probability sampling. After excluding empty or non-resident dwellings, final interviews were conducted on 17708 respondents from 10257 households, which completed at least one module of the survey beyond the cover screening for age eligibility. CHARLS respondents were followed every 2 years, using a face-to-face computer-assisted personal interview (CAPI) ${ }^{13}$ SHS mainly affects married women in China. Although unmarried or cohabiting women can possibly be affected by household SHS, this kind of influence remains scarce. At baseline, there were 3381 married women who never smoked cigarettes and lived with spouses who had either smoked cigarettes in the past or smoked at the time of the interview. Data for each variable were therefore collected for those respondents. Our final sample was composed of 6875 respondents. Among them, 2802 were interviewed again during the second wave of data collection in 2013, and 2247 were interviewed again during the third wave in 2015 . The similar sample selection process was conducted for participants in the second wave in 2013 as a baseline. The final sample consisted of 1799 women who were investigated again in 2015 as participants.

\section{Measures}

Secondhand smoke

In this study, exposure to SHS among Chinese women was assessed through several surveys based on the standardised CHARLS questionnaire. Questions about participants' current marital status, the year they got married and the year the husband in each household had begun or ceased smoking at home were asked.

The smoking status section contained four questions: 'Have you ever chewed tobacco, smoked a pipe, smoked self-rolled cigarettes or smoked cigarettes/cigars?' 'Do you still have the habit or have you totally quit?' 'At what age did you totally quit smoking?' and 'At what age did you start to smoke on a regular basis?' If the answer to the first question was 'yes', they were defined as 'current smokers' or 'ex-smokers'. Our analysis of SHS exposure focused only on never-smokers, excluding the 'current smokers' and 'ex-smokers', because of the difficulty to differentiate the negative effects of active smoking on health condition from those of SHS exposure. The length of SHS exposure was calculated and expressed as the total number of years that never-smoking women spent living with their spouses who smoked cigarettes at home.

Categorical classification of SHS was used because the impact of SHS might be neglected if we only used a continuous variable to represent exposure. Compared with continuous variables, categorical variables have greater public health significance. Based on the constructed SHS exposure variable, participants were classified into four different groups: never or being exposed to SHS for $<25$ years, $\geq 25$ years and $<30$ years, $\geq 30$ years and $<40$ years, and $\geq 40$ years. The cut-off boundaries of SHS exposure were decided to realise the relatively balanced population 
distribution frequency among different levels of exposure year.

\section{Cognitive function}

The cognitive function of the respondents in CHARLS questionnaires was measured through a question-andanswer interview instrument, and the respondents were followed every 2 years using a face-to-face CAPI. The various sections of the questionnaire could assess cognitive subdomains including visuospatial ability, orientation and attention, and episodic memory. Figure drawing was tested by asking the participants to reproduce a picture of two overlapped pentagons in CHARLS questionnaires. ${ }^{17}$ It was used to measure a person's ability to identify visual and spatial relationships among objects. The Telephone Interview of Cognitive Status (TICS) was a screening test, including serial subtractions of 7 from 100 (up to five times), date (month, day, and year and season) and the day of the week. To assess orientation and attention function, the number of correct answers to the above questions in TICS was scored and summed up (range, 0-10). Participants who successfully completed the task received a score of 1 , and those who failed received a score of $0 .{ }^{18}$

In addition, the word recall test consisted of two components, immediate recall and delayed recall, and evaluated episodic memory. Participants were required to repeat 10 Chinese nouns just read to them, and then after 20 questions concerning Center for Epidemiologic Studies Depression Scale (CES-D; approximately 4-10 min) they were again asked to recall as many of the original words as possible. The item was coded as 1 if recalled by the respondent and as 0 if not. Scores for both immediate and delayed recall varied from 0 to 10 . An evaluated episodic memory score was calculated using the mean of scores in immediate and delayed word recall (range, $0-10){ }^{18}$

The overall cognition scores were the sum of the three different domains (range, 0-21).

\section{Control variables}

Given that cognitive function may vary across demographic and socioeconomic status, we thus included age, urban/rural residence, education, annual household expenditures, chronic diseases and depressive symptoms as control variables. Education was categorised into three groups: 'illiterate', 'primary education' and 'secondary education or above'. Arterial hypertension and diabetes mellitus are separately strong independent risk factors for the development of cognitive impairment and dementia. ${ }^{190}$ Thus, the baseline chronic disease of hypertension and diabetes was classified into three types based on self-reported conditions on whether the participants were being treated: having hypertension/diabetes with treatment, having hypertension/diabetes without treatment and not having hypertension/diabetes. The measure of depressive symptoms was based on the 10-item version of the CES-D short form, and each of the fouroption response to the item was scored ranging from 0 to 3 . The total score is the sum of points for all 10 items, and a score of 10 or higher suggests the presence of depressive symptoms. ${ }^{6}$

\section{Patient and public involvement}

Patients and/or the public were not involved in the design, or conduct, or reporting, or dissemination plans of this research.

\section{Analysis}

All analyses were conducted with STATA V.14.0 (Stata, College Station, Texas, USA). Lagged dependent variable (LDV) regression models with ordinary least squares estimation were used during analysis. LDV models were superior for analysing the effects of predictor variables on an outcome with two-wave panel data while controlling for the influence of time-invariant variables. ${ }^{21}$ It adjusted for baseline cognitive conditions for all participants, thereby providing more robust estimates of the effects of independent variables. After pooling the three sets of panel data into one through using the 'year' dummy variable to differentiate between a change in 2 years and 4 years, we have 6875 respondents who have complete data on all variables. The overall cognitive scores, episodic memory scores, visuospatial ability scores, and orientation and attention scores were four separate outcome variables. The different groups of SHS exposure years were the predictor variable, and other independent variables included all demographic and socioeconomic characteristics. Prior to fitting the regression models, descriptive analyses were conducted to estimate the mean and SD for continuous data and frequencies and percentages for categorical data.

\section{RESULTS}

Table 1 provides a descriptive summary of all the variables for participants from each panel of three different waves: 2011-2013, 2011-2015 and 2013-2015. High prevalence of SHS exposure between those aged 30 and 40 years was seen in different panels, accounting for $32.51 \%, 35.18 \%$ and $42.69 \%$, respectively.

The participants were $>45$ years old, with a mean age of 56, 56 and 58 years, respectively, in those waves. Participants were more likely to live in a rural area, have a lower education background and not have a hypertension or diabetes diagnosis at baseline. In addition, our results indicated that the mean baseline cognition scores were higher than the cognition scores after 2 years or 4 years. The mean scores of CES-D suggested that the prevalence of depression among Chinese middle-aged and old-aged women increased in those years. Other sociodemographic characteristics of the respondents are shown in table 1

Results from the regression models for the relationship between SHS exposure and each domain of the cognitive function and overall cognition scores are reported in tables 2 and 3. Scores of episodic memory, orientation and attention, and visuospatial ability among respondents at baseline were strong predictors of their 


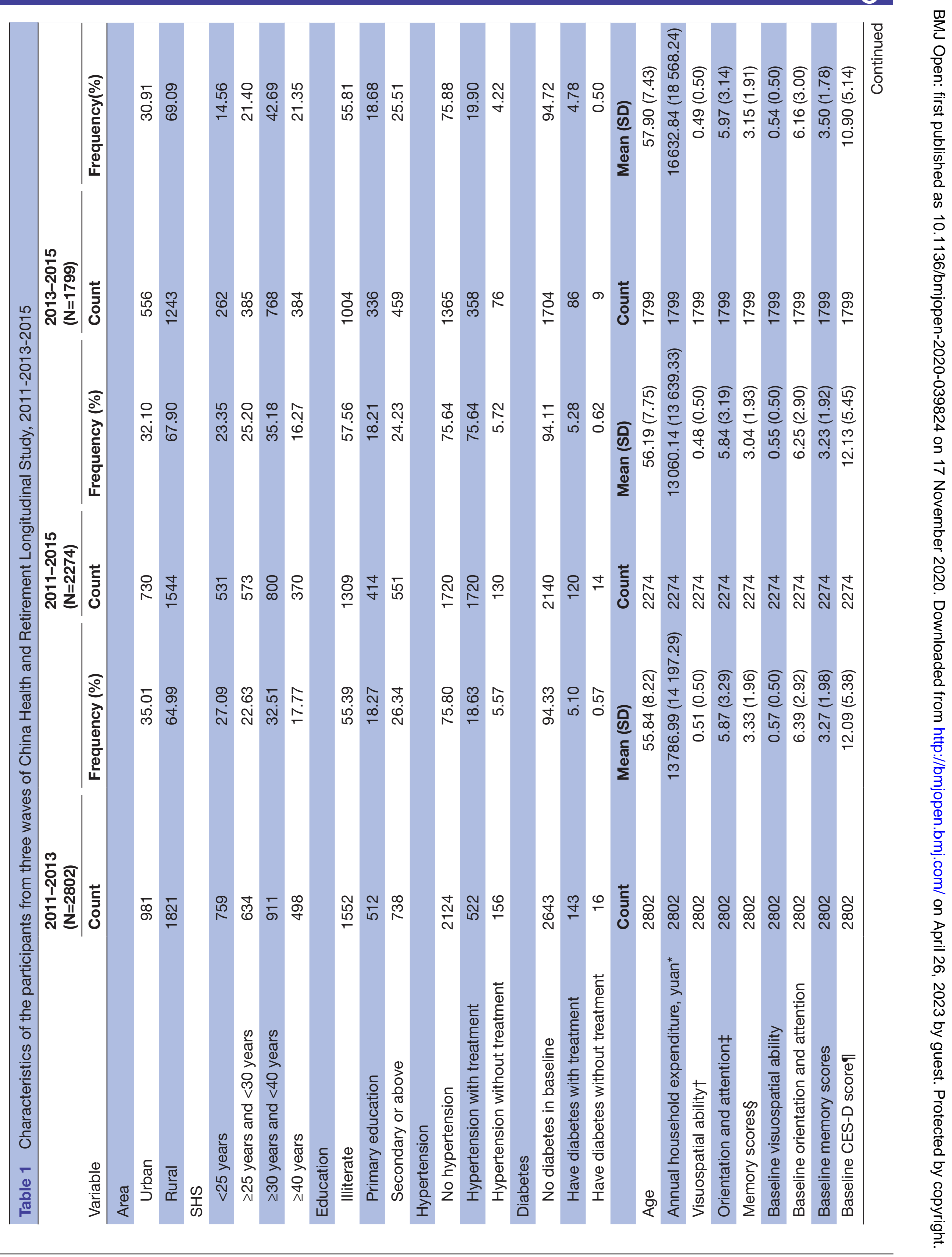




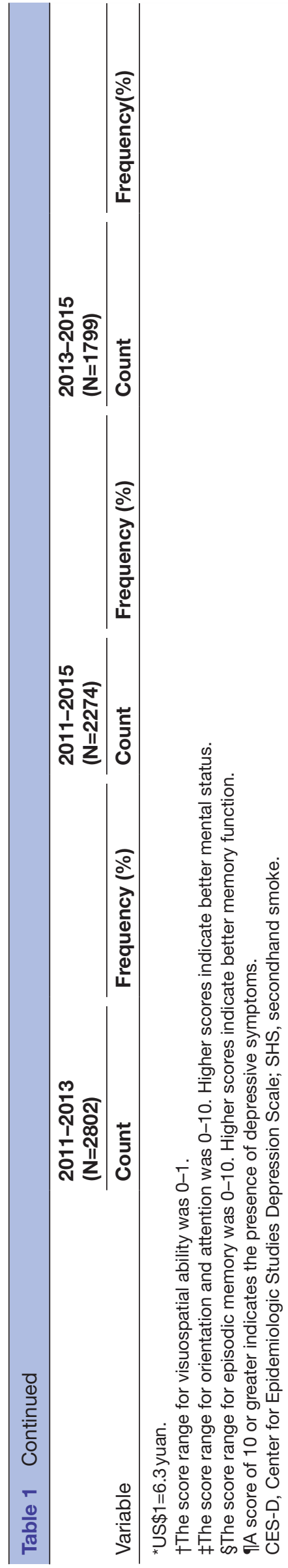

corresponding cognitive function measures after 2 years or 4 years. Based on the analysis adjusted for age, annual household expenditure, education, baseline cognitive function and another chronic health status, we found that only being exposed to SHS for more than 40 years was significantly associated with a decline in visuospatial abilities, episodic memory and overall cognition scores for all respondents. Compared with respondents who were not exposed to SHS or exposed to it for less than 25 years, those who have been exposed to SHS for more than 40 years was associated with a 0.04-point decline in visuospatial abilities $(95 \% \mathrm{CI}=-0.08$ to $-0.01, \mathrm{p}<0.1)$, a 0.16 -point decline in episodic memory $(95 \% \mathrm{CI}=-0.31$ to $-0.01, \mathrm{p}<0.05)$, and a 0.33-point decline in overall cognition function $(95 \% \mathrm{CI}=-0.66$ to $-0.01, \mathrm{p}<0.01)$. In addition, age was also negatively associated with cognitive function. Each 1 year increase was associated with a 0.01-point, 0.01-point, 0.03-point and 0.05-point decrease in visuospatial $(95 \% \mathrm{CI}=-0.01$ to $-0.00, \mathrm{p}<0.01)$, orientation $(95 \% \mathrm{CI}=-0.03$ to $-0.01, \mathrm{p}<0.01)$, memory $(95 \% \mathrm{CI}=-0.31$ to $-0.01, \mathrm{p}<0.05)$ and overall cognition scores $(95 \% \mathrm{CI}=-0.66$ to $-0.01, \mathrm{p}<0.01)$, respectively. High education level was associated with better cognitive performance, especially in orientation and attention. In addition, a 1-point increase in CES-D scores was associated with a 0.02-point decrease in scores of orientation and attention $(95 \% \mathrm{CI}=-0.03$ to $0.00, \mathrm{p}<0.05)$, showing that respondents with depressive symptoms were more likely to demonstrate a cognitive decline in specific functions.

\section{DISCUSSION}

Results from this longitudinal study with a large, representative sample of middle-aged and older women in China indicated that exposure to SHS for more than 40 years was significantly associated with declining performance of global cognition and cognitive subdomains. It is the first examination of cognitive subdomains concerning household SHS exposure using a 4-year longitudinal data in China. The inferior performance of SHS on visuospatial abilities, episodic memory, and orientation and attention abilities is novel because these domains were not specifically evaluated in earlier studies among middle-aged and older women who never smoke. This study builds on previous findings that SHS was associated with poor cognitive performance, especially in children, adolescents and adults. ${ }^{17}$ We found that having a high educational level, living in an urban area and having better baseline cognitive function would improve their cognitive performance. The episodic memory score of participants with diabetes at baseline decreased by 0.172 points compared with those without diabetes, which is similar to previous findings. ${ }^{9}$

Moreover, our results showed that compared with women who have never been exposed to SHS or have been exposed for less than 20 years, those who have been exposed to SHS for more than 40 years have a significant decline in visuospatial function (0.04 points), episodic 
Table 2 Adjusted multivariable linear regression analysis of the relationship between smoking exposure and visuospatial function and orientation and attention among older Chinese women (N=6875), 2011-2013-2015

\begin{tabular}{|c|c|c|c|c|c|c|}
\hline \multirow[b]{2}{*}{ Variables } & \multicolumn{3}{|c|}{ Visuospatial function } & \multicolumn{3}{|c|}{ Orientation and attention } \\
\hline & $\beta$ coefficient & $95 \% \mathrm{Cl}$ & $\mathbf{t}$ & $\beta$ coefficient & $95 \% \mathrm{Cl}$ & $\mathbf{t}$ \\
\hline Age & $-0.01^{*}$ & -0.01 to 0.00 & -6.73 & $-0.02^{*}$ & -0.03 to -0.01 & -4.34 \\
\hline \multicolumn{7}{|l|}{ SHS exposure } \\
\hline$\geq 25$ years to $<30$ years & -0.01 & -0.04 to 0.02 & -0.72 & 0.05 & -0.12 to 0.21 & 0.56 \\
\hline Baseline visuospatial function & $0.23^{*}$ & 0.21 to 0.26 & 17.93 & & & \\
\hline Baseline orientation and attention & & & & $0.55^{\star}$ & 0.53 to 0.57 & 44.86 \\
\hline Urban§ & $0.06^{*}$ & 0.04 to 0.09 & 5.27 & $0.38^{*}$ & 0.25 to 0.51 & 5.72 \\
\hline \multicolumn{7}{|l|}{ Educationף } \\
\hline With treatment & -0.03 & -0.09 to 0.02 & -1.20 & 0.06 & -0.24 to 0.35 & -0.38 \\
\hline Without treatment & -0.02 & -0.06 to 0.03 & -0.69 & 0.06 & -0.11 to 0.24 & 0.04 \\
\hline Missing group & $-0.05 \ddagger$ & -0.12 to 0.01 & -1.67 & 0.06 & -0.19 to 0.30 & -0.01 \\
\hline \multicolumn{7}{|l|}{ Diabetest† } \\
\hline With treatment & 0.02 & -0.03 to 0.07 & 0.90 & $0.25 \ddagger$ & -0.03 to 0.52 & -1.77 \\
\hline Without treatment & 0.03 & -0.04 to 0.09 & 0.82 & 0.21 & -0.06 to 0.48 & -0.23 \\
\hline Missing group & 0.07 & -0.08 to 0.21 & 0.88 & 0.50 & -0.22 to 1.23 & 0.67 \\
\hline Baseline CES-D score & $-0.00 \ddagger$ & -0.00 to 0.00 & -1.71 & -0.02 & -0.03 to -0.00 & -2.57 \\
\hline Year=2 & -0.02 & -0.04 to 0.01 & -1.58 & 0.09 & -0.05 to 0.22 & 1.29 \\
\hline
\end{tabular}

${ }^{*} \mathrm{p}<0.01$.

†Reference: No SHS exposure or $<25$ years.

$\ddagger \mathrm{p}<0.1$.

$\S$ Expenditure is expressed as the natural log of the annual household expenditure.

IReference: Illiterate.

${ }^{* *}$ Reference: Without hypertension.

††Reference: Without diabetes.

$\ddagger \ddagger p<0.05$.

$\S \S$ This model adjusted for age, expenditure, living area, education, baseline hypertension, diabetes, depression status and baseline cognitive function.

CES-D, Center for Epidemiologic Studies Depression Scale; SHS, secondhand smoke.

memory (0.16 points) and overall cognitive scores $(0.33$ points). These findings were similar in magnitude to prior research on the relationship between SHS and cognitive function. ${ }^{13}$ Moheet and colleagues conducted a crosssectional study in the North East of England to explore the impact of diabetes on cognitive function and brain structure $(\mathrm{N}=150)$. Research suggested that compared with non-exposed people, participants who had no history of smoking and were being averagely exposed to SHS for around 6 years showed significantly reduced performance in processing speed (ie, how quickly one can process information and perform tasks) and executive function (ie, the ability to organise memory, cognitive flexibility and problem-solving ability). ${ }^{21}$ Another longitudinal ageing study in China on those aged $\geq 50$ years $(\mathrm{N}=4809)$ found that never-smokers exposed to the highest levels of SHS (salivary cotinine concentrations of $0.8-13.5 \mathrm{ng} / \mathrm{mL}$ ) were more likely to be cognitively impaired $(\mathrm{OR}=1.70)$ than those exposed to little or no SHS. ${ }^{17}$

Attention, which referred to the ability to concentrate and focus on specific stimuli, slightly declined in later life. ${ }^{12}$ Orientation was one's ability to identify the exact date, month, day and season of the year. ${ }^{22}$ Our results suggested that for each 1 year increase in age, there was an additional 0.01-point, 0.02-point, 0.04-point and 0.06 -point decline in visuospatial, orientation, memory and overall cognition scores, respectively. SHS seems to be more strongly associated with cognitive decline than ageing, since the magnitude of significant coefficient between SHS and cognitive decline was almost four times the one in ageing. However, the relationship between SHS and orientation and attention ability was not observed. This may be because the size of the sample is relatively 
Table 3 Adjusted multivariable linear regression analysis of the relationship between smoking exposure and episodic memory and overall cognitive function among older Chinese women ( $N=6875), 2011-2013-2015$

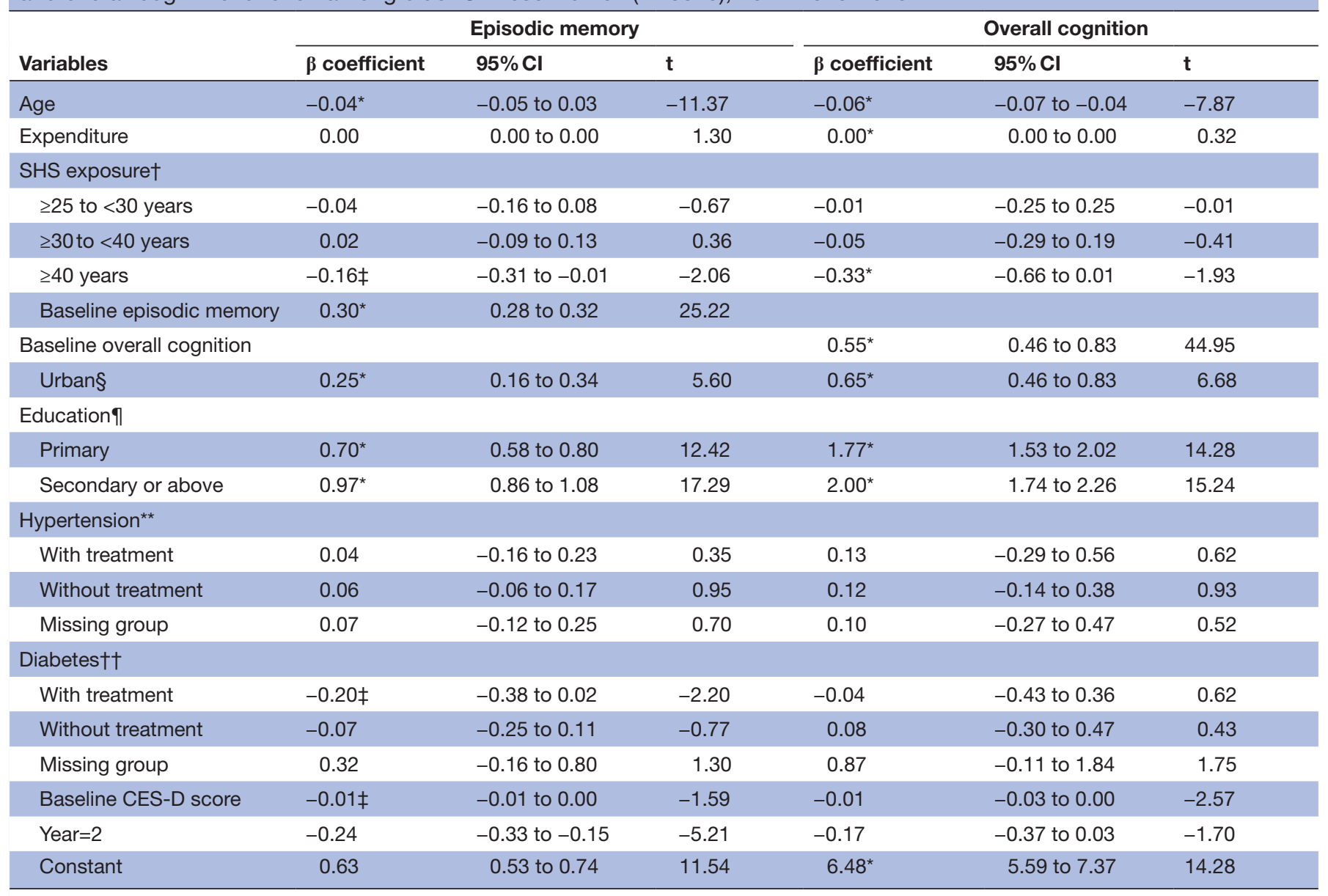

${ }^{*} \mathrm{p}<0.01$.

†Reference: No SHS exposure or $<25$ years.

$\neq \mathrm{p}<0.05$.

$\S$ Expenditure is expressed as the natural log of the annual household expenditure.

qReference: Illiterate.

${ }^{\star *}$ Reference: Without hypertension.

††Reference: Without diabetes.

$\ddagger \ddagger \mathrm{p}<0.1$.

$\S \S$ This model adjusted for age, expenditure, living area, education, baseline hypertension, diabetes, depression status and baseline cognitive function.

CES-D, Center for Epidemiologic Studies Depression Scale; SHS, secondhand smoke.

small, plus the period of cohort study after controlling for all demographic and socioeconomic confounders is relatively short.

Visuospatial abilities involve the ability to understand space in two and three dimensions. In our study, an inversed relationship between SHS exposure and visuospatial abilities among middle-aged and older adults was observed, showing a 0.04-point decline in their visuospatial scores. Such an inversed relationship between SHS exposure and visuospatial reasoning skills was also reported among American children aged 6-16 years $(\mathrm{N}=5683)$, showing that years of SHS exposure was significantly associated with lower scores for reading, mathematics and visuospatial skills, after adjusting for covariates. ${ }^{14}$ As one of the most common cognitive complaints among elders, episodic memory refers to personally experienced events that could be measured by stories, word lists or figures. Previous research has indicated that the onset of memory decline may vary among different memory types, with episodic memory decline possibly being lifelong. ${ }^{23}$ Our study could not explore the onset age of memory decline without performing regression among different age groups. The significant coefficient may indicate memory decline associated with SHS exposure.

The inconsistent conclusions between our studies and prior ones may be due to the relatively simplified version of the cognition test procedure in CHARLS questionnaires compared with the Montreal Congnitive Assessment (MoCA) ${ }^{13}$ and Mini-Mental State Examination. ${ }^{24}$ Some studies also used clinical or MRI evidence of neurological damage to detect cognitive impairment. The MoCA functions best as a screening test, having exhibited 
excellent sensitivity in identifying MCI and Alzheimer's disease $(\mathrm{AD})$ at $90 \%$ and $100 \%$, respectively. ${ }^{25}$

The most popular hypothesis about the mechanisms underlying the links between SHS exposure and more unsatisfactory cognitive performance lies in the notion that the carbon monoxide in tobacco smoke may interfere with the oxygen being delivered to the brain via the blood. However, the reasons behind the different effects on various domains of brain function are far from clear. One possible explanation derives from research on laboratory animals. Exposing animals to varying degrees of toxic mixtures of chemicals found in tobacco smoke may lead to reduced neuronal mass in specific regions of the brain associated with learning and memory. Since the hippocampal region of the brain is known to be involved in the mediation of memory ${ }^{26}$ and learning, further research should be conducted in other regions dominating visuospatial and orientation ability. Another possible mechanism is that prolonged exposure to SHS may be a significant risk factor for cardiovascular disease (CVD),${ }^{27}$ which may therefore lead to a range of health and cognitive problems in later life. In the future, a longitudinal design may elucidate any associations by observing long-term exposure to SHS and the incidence of CVD, and whether this CVD may mediate or interact with SHS exposure to impact cognitive function.

Several limitations need to be considered when interpreting this study and designing future studies. First, exposure to SHS was evaluated based on self-report measures. This might be subject to recall bias and lead to overestimation or underestimation of exposure. ${ }^{28}$ Therefore, further studies could include more biological assays, for example, cotinine residue levels or nicotine residue in saliva or hair samples. ${ }^{29}$ Previous research using serum cotinine as a biomarker of exposure to SHS found that higher levels of serum cotinine were associated with significantly worse performance in reading, mathematics, and visual and spatial abilities in children and adolescents. ${ }^{8}$ However, no studies have used a combination of biomarker and self-reporting yet. ${ }^{30}$ Some validated biomarkers could be used as proxies for $\mathrm{AD}$ neuropathological changes, such as cerebrospinal fluid amyloid-beta $(A \beta) 42$ concentrations or $A \beta 42 / A \beta 40$ ratio and amyloid load on positron emission tomography scans. These biomarkers could provide more reliable measures of cognitive impairment. ${ }^{31}$ Second, it may be impossible to control for all potentially confounding variables. After adjusting for age, household expenditure, education, area, chronic health condition and depressive symptoms, some other demographic or socioeconomic confounders may still have been neglected. However, this did not appear to affect the magnitude of the association between SHS exposure and cognition. ${ }^{13}$ Besides, the analyses only contained household SHS exposure, which precluded the analyses of the influence of environmental smoke inhalation on smoking proclivity. Whether exposure to household SHS can hasten the onset of cognitive impairment for older Chinese women could be further proved by running regression models in different age groups.

Acknowledgements We would like to thank Professor Yaohui Zhao at Centre for Chinese Economic Research, Peking University and Mr Benjamin Martin at Birmingham Women's and Children's Hospital for their thoughtful contributions to this study.

Contributors $A B$ wrote and participated in all aspects of this research, including the field investigation. YH reviewed and supervised this research. YJ participated in the statistical analysis of this work and reviewed the final article.

Funding This is a self-funded research.

Competing interests None declared.

Patient consent for publication Not required.

Provenance and peer review Not commissioned; externally peer reviewed.

Data availability statement The data are available at http://charls.pku.edu.cn.

Open access This is an open access article distributed in accordance with the Creative Commons Attribution Non Commercial (CC BY-NC 4.0) license, which permits others to distribute, remix, adapt, build upon this work non-commercially, and license their derivative works on different terms, provided the original work is properly cited, appropriate credit is given, any changes made indicated, and the use is non-commercial. See: http://creativecommons.org/licenses/by-nc/4.0/.

ORCID iDs

Yinzi Jin http://orcid.org/0000-0003-0634-3955

Yangmu Huang http://orcid.org/0000-0002-3660-1276

\section{REFERENCES}

1 Fang EF, Scheibye-Knudsen M, Jahn HJ, et al. A research agenda for aging in China in the 21st century. Ageing Res Rev 2015;24:197-205.

2 Harada CN, Natelson Love MC, Triebel KL. Normal cognitive aging. Clin Geriatr Med 2013;29:737-52.

3 Robertson DA, Savva GM, Kenny RA. Frailty and cognitive impairment--a review of the evidence and causal mechanisms. Ageing Res Rev 2013;12:840-51.

4 Yin $\mathrm{P}, \mathrm{Ma} \mathrm{Q}$, Wang $\mathrm{L}$, et al. Chronic obstructive pulmonary disease and cognitive impairment in the Chinese elderly population: a large national survey. Int J Chron Obstruct Pulmon Dis 2016;11:399.

5 Bernardin F, Maheut-Bosser A, Paille F. Cognitive impairments in alcohol-dependent subjects. Front Psychiatry 2014;5:78.

6 Tadic M, Cuspidi C, Hering D. Hypertension and cognitive dysfunction in elderly: blood pressure management for this global burden. BMC Cardiovasc Disord 2016;16:208.

7 Modrego PJ, Ferrández J. Depression in patients with mild cognitive impairment increases the risk of developing dementia of Alzheimer type: a prospective cohort study. Arch Neurol 2004;61:1290-3.

8 Ling J, Heffernan T. The cognitive deficits associated with secondhand smoking. Front Psychiatry 2016;7:46.

9 Durazzo TC, Meyerhoff DJ, Nixon SJ. A comprehensive assessment of Neurocognition in middle-aged chronic cigarette smokers. Drug Alcohol Depend 2012;122:105-11.

10 Teo KK, Ounpuu S, Hawken S, et al. Tobacco use and risk of myocardial infarction in 52 countries in the INTERHEART study: a case-control study. Lancet 2006;368:647-58.

11 Kim BJ, Kang JG, Kim JH, et al. Association between Secondhand smoke exposure and hypertension in 106,268 Korean self-reported never-smokers verified by cotinine. J Clin Med 2019;8:1238.

12 Heffernan TM, O'Neill TS. Everyday prospective memory and executive function deficits associated with exposure to second-hand smoke. J Addict 2013;2013:1-7.

13 Yolton K, Dietrich K, Auinger P, et al. Exposure to environmental tobacco smoke and cognitive abilities among U.S. children and adolescents. Environ Health Perspect 2005;113:98-103.

14 Llewellyn DJ, Lang IA, Langa KM, et al. Exposure to secondhand smoke and cognitive impairment in non-smokers: national cross sectional study with cotinine measurement. BMJ 2009;338:b462

15 Malek AM, Cushman M, Lackland DT, et al. Secondhand smoke exposure and stroke: the reasons for geographic and racial differences in stroke (regards) study. Am J Prev Med 2015;49:e89-97.

$16 \mathrm{He} \mathrm{F}$, Li T, Lin J, et al. Passive smoking exposure in living environments reduces cognitive function: a prospective cohort study in older adults. Int J Environ Res Public Health 2020;17:1402. 
17 Pan X, Luo Y, Roberts AR. Secondhand smoke and women's cognitive function in China. Am J Epidemiol 2018;187:911-8.

18 Zhao Y, Hu Y, Smith JP, et al. Cohort profile: the China health and retirement longitudinal study (CHARLS). Int J Epidemiol 2014;43:61-8.

19 Ge S, Wei Z, Liu T, et al. Alcohol use and cognitive functioning among middle-aged and older adults in China: findings of the China health and retirement longitudinal study baseline survey. Alcohol Clin Exp Res 2018;42:2054-60.

$20 \mathrm{Li} \mathrm{J}$, Cacchione PZ, Hodgson N, et al. Afternoon napping and cognition in Chinese older adults: findings from the China health and retirement longitudinal study baseline assessment. J Am Geriatr Soc 2017;65:373-80.

21 Moheet A, Mangia S, Seaquist ER. Impact of diabetes on cognitive function and brain structure. Ann N Y Acad Sci 2015;1353:60-71.

22 Peters-Founshtein G, Peer M, Rein Y, et al. Mental-orientation: A new approach to assessing patients across the Alzheimer's disease spectrum. Neuropsychology 2018;32:690-9.

23 Burk JA, Blumenthal SA, Maness EB. Neuropharmacology of attention. Eur J Pharmacol 2018;835:162-8.

24 Lövdén M, Rönnlund M, Wahlin A, et al. The extent of stability and change in episodic and semantic memory in old age: demographic predictors of level and change. J Gerontol B Psychol Sci Soc Sci 2004;59:P130-4.
25 Li X, Jia S, Zhou Z, et al. The role of the Montreal cognitive assessment (MoCA) and its memory tasks for detecting mild cognitive impairment. Neurol Sci 2018;39:1029-34.

26 Trzepacz PT, Hochstetler H, Wang S, et al. Relationship between the Montreal cognitive assessment and Mini-Mental state examination for assessment of mild cognitive impairment in older adults. BMC Geriatr 2015;15:107.

27 Ziad S, Nasreddine M. The Montreal cognitive assessment (MoCA)concept and clinical review. J Am Geriatr Soc 2005;53:695-9.

28 Staples MC, Mandyam CD. Thinking after drinking: impaired hippocampal-dependent cognition in human alcoholics and animal models of alcohol dependence. Front Psychiatry 2016;7:162.

29 Yankelevitz DF, Henschke Cl, Yip R, et al. Second-hand tobacco smoke in never smokers is a significant risk factor for coronary artery calcification. JACC Cardiovasc Imaging 2013;6:651-7.

30 Akhtar WZ, Andresen EM, Cannell MB, et al. Association of blood cotinine level with cognitive and physical performance in nonsmoking older adults. Environ Res 2013;121:64-70.

31 Banning LCP, Ramakers IHGB, Deckers K, et al. Affective symptoms and $A T(N)$ biomarkers in mild cognitive impairment and Alzheimer's disease: A systematic literature review. Neurosci Biobehav Rev 2019;107:346-59. 